\title{
Anomalies in the WordNet Verb Hierarchy
}

\author{
Tom Richens \\ Aston University \\ Aston Triangle \\ Birmingham B4 7ET, England \\ richentmeaston.ac.uk
}

\begin{abstract}
The WordNet verb hierarchy is tested, with a view to improving the performance of its applications, revealing topological anomalies and casting doubt on its semantic categories. Encoded troponyms frequently misrepresent other kinds of entailment. Approaches are proposed for correcting these anomalies including a new top ontology.
\end{abstract}

\section{Introduction}

WordNet is a lexical database widely used for NLP tasks. Any application of WordNet which measures semantic distance employs WordNet relations to do so.

As part of a wider project to build an improved and enriched lexical database using existing resources, in the hope of improving on the performance of WordNet, this study investigates the correctness of the hypernymy/troponymy relations between verbs.

The broader project would ideally model these hierarchical relations as trees but for the phenomenon of multiple inheritance, which is investigated here to see if it is semantically justified. Moreover it seems intuitively likely that anomalies will be concentrated where the relational structure is more complex.

\subsection{Definitions}

The only document found to specify the WordNet verbal relations is Fellbaum (1998), who defines troponymy (the verb equivalent of $h y$ ponymy) as a special case of entailment, where

(C) 2008. Licensed under the Creative Commons Attribution-Noncommercial-Share Alike 3.0 Unported license (http://creativecommons.org/licenses/by-nc$\underline{\mathrm{sa} / 3.0 /)}$. Some rights reserved. the entailing and entailed verb are temporally coextensive, distinguishing it from causation, temporal inclusion, and backward presupposition, of which the last two are not encoded as distinct relations in WordNet, but are combined under the general heading of entailment. The expression sister terms is used to designate a pair of synsets which share a hypernym.

This study distinguishes between transitive causation (causing another to do something) and reflexive causation (causing oneself to do something). The term quale (plural: qualia) is borrowed from Pustejovsky (1991). As applied to verbs within the context of this study, the formal quale means what is physically done, while the telic quale means the purpose or intended result of the action.

\subsection{Application of WordNet Relations}

Banerjee \& Pedersen (2003) have employed WordNet relations in an extension to the Lesk (1986) algorithm for word sense disambiguation. In order to establish the relatedness of two words, the glosses of their WordNet relatives are compared. Their results are noticeably inferior for verbs than for nouns. Moreover, while the most useful relations for disambiguating nouns were hyponymy and meronymy, in the case of verbs, the example sentences proved more useful than any relations. Their best results were obtained by using all relations between verbs indiscriminately. This finding reflects on the distinctions between kinds of verb relations and suggests that at least where verbs are concerned, the limited success achieved by algorithms relying on WordNet relations arises from the probability that when a relation is encoded, some relation exists, even though the kind of relation is not necessarily correct. This observation suggests that improvements to the WordNet relations may well be useful for improving on the performance 
of WordNet as a tool for interlingual tasks and word sense disambiguation.

\subsection{Validation of Wordnets}

Smrž (2003) proposes a number of tests for validating wordnets. These involve checking for "cycles", "dangling uplinks" and "top-most synset not from the defined set" and critically examining "multi-parent relations". These tests have been applied in the development of BalkanNet (Various 2004). The present study looks for any of the listed faults or potential faults within WordNet 3.0. Without a defined set of unique beginners, it is impossible to distinguish a "dangling uplink" from "top-most synset not from the defined set". All verbs synsets without any hypernym will therefore be considered as candidate unique beginners.

\subsection{Potential Hypernymy Faults}

Liu et al. (2004) have found thousands of cases of rings within the hierarchies, which arise when a synset has two hypernyms within the same semantic category, which themselves have a common hypernym, along with isolators, trees isolated within their own category whose only hypernym lies in another category. Such deviations from a tree-structure, if not semantically justifiable, should be considered as faults.

The occurrence of a ring implies multiple inheritance, which is not necessarily a fault but merits investigation to ensure that it is justified. There are two other kinds of potential fault which should also be considered: another kind of ring is a cycle, which is formed where following the hypernymy relation in one direction leads back to where one started; a special case of an isolator occurs where a synset has no hypernym at all, which makes it a candidate unique beginner. Liu et al. (2004) dismiss this possibility as legitimate, without further consideration, on the grounds that this applies to the unique beginners of each semantic category. In fact, Fellbaum (1998) allows for more than one unique beginner per verb category. However cases where there is a large number of unique beginners in one category merit investigation.

\subsection{Methodology}

The present study has been conducted using a model of WordNet where synsets, word senses and relations are implemented as Java objects, constructed from the WordNet 3.0 Prolog files. Focussing mainly on verbs, three main aspects are investigated, topological anomalies, anomalies relating to categories and the top ontology.

An algorithm was developed to discover occurrences of each of the kinds of potential hypernymy fault identified above. To investigate topological anomalies, the algorithm recursively constructs an upside-down tree from each synset, using that synset as root with its most remote indirect hypernyms as the leaves. Where a cycle occurs, a stack error will result. A ring is identified wherever a synset is found more than once in the same upside-down tree. This approach is an extension of the methodology employed by Liu et al. (2004), in that it assumes no correlation between semantic categories and hypernymy so as to identify rings which straddle category boundaries. An examination of rings in the verb hierarchy leads onto a more general examination of dual inheritance among verbs (there are no verbs with more than 2 hypernyms).

An isolator occurs when the only hypernym is in a different semantic category to the synset under investigation. Examination of isolators reveals more anomalies in the verb hierarchy but also raises questions about the validity of the semantic categories.

A candidate unique beginner is identified whenever a synset has troponyms but no hypernym. The proliferation of unspecified unique beginners among verbs prompts a full review of the top ontology.

The full results which are analysed here are currently available at:

http://www.rockhouse.me.uk/Linguistics.

\section{Topological Anomalies and Remedies}

\subsection{Cycle}

The algorithm implementation generated a stack error when applied to a number of verbal synsets: in each case the same cycle was encountered, which is the only one in WordNet 3.0.

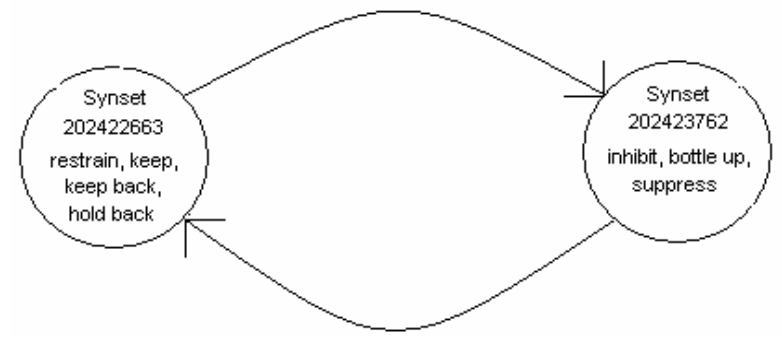

Figure 1. Cycle topology

The two synsets in fig. 1 appear to be synonymous. Merging them to remove the cycle would improve structural consistency. 


\subsection{Rings}

Liu et al. (2004) define a ring as being formed where a synset "has at least 2 fathers in its own category", which have a common ancestor also within that category. Our algorithm uses a broader definition of ring as any instance of a synset having two hypernyms such that these hypernyms themselves have a common ancestor or one of them is the ancestor of the other (table $1)$.

\begin{tabular}{|l|l|l|}
\hline $\begin{array}{l}\text { Case with respect to } \\
\text { semantic categories }\end{array}$ & Verbs & Nouns \\
\hline Single category & 5 & 1 \\
\hline $\begin{array}{l}\text { Ancestry crosses categories } \\
\text { but direct relations are in } \\
\text { same category as headword }\end{array}$ & 2 & 1984 \\
\hline $\begin{array}{l}\text { Ancestry crosses categories } \\
\text { and direct relations cross } \\
\text { categories }\end{array}$ & 1 & 379 \\
\hline TOTAL & $\mathbf{8}$ & $\mathbf{2 3 6 4}$ \\
\hline $\begin{array}{l}\text { TOTAL using definition } \\
\text { from Liu et al. (2004) }\end{array}$ & $\mathbf{7}$ & $\mathbf{1 9 8 5}$ \\
\hline $\begin{array}{l}\text { Results of Liu et al. (2004) } \\
\text { using WordNet 2.0 }\end{array}$ & $\mathbf{1 7}$ & $\mathbf{1 8 3 9}$ \\
\hline
\end{tabular}

Table 1. Rings in Wordnet

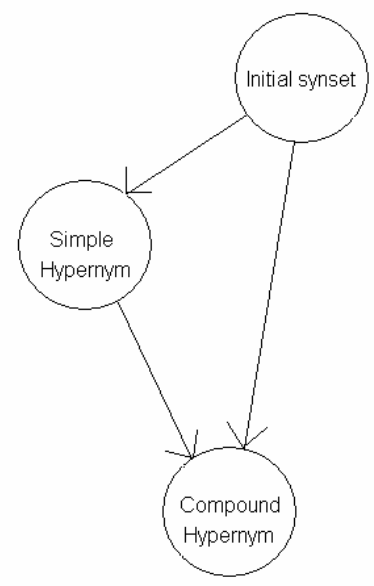

Figure 2. Asymmetric ring topology

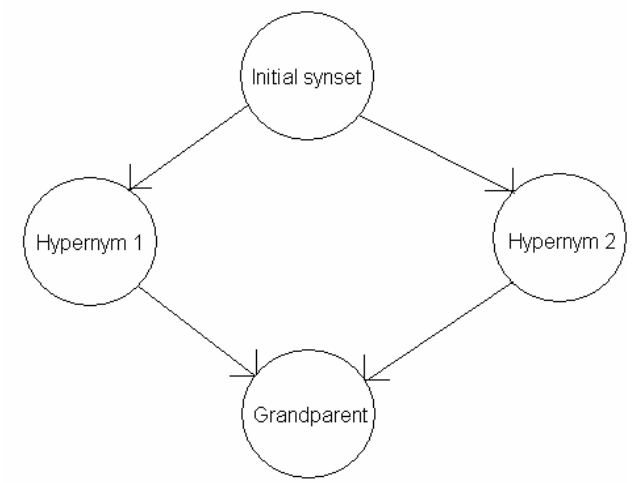

Figure 3. Symmetric ring topology
An analysis of the rings among nouns is outside the scope of this study. Out of the 8 rings in the verb hierarchies, 4 are asymmetric and 4 are symmetric.

In 3 out of 4 cases of the asymmetric topology (fig. 2), the link between the initial synset and the compound hypernym is redundant and can be removed. In the remaining case, "eat" (transitive) has the simple hypernym "eat" (intransitive) which simply fails to specify what is eaten. This can be considered as synonymy and so there is a case for merging these synsets. The compound hypernym "consume, ingest" would also seem to be a synonym, unless one relies on the gloss: "serve oneself to, or consume regularly", in which case it as ambiguous between reflexive causation and iteration, neither of which fits the definition of hypernymy.

The instances of symmetric topology (fig. 3) raise different issues. Liu et al. (2004) assert that a ring implies a paradox because they assume that two hyponyms of a single hypernym must have opposite properties in some dimension and therefore cannot have a common hyponym, as a hyponym must inherit all the properties of its hypernym. In fact there need not be any paradox as two hyponyms can modify two different properties of their hypernym (see Amaro et al., 2006, for a discussion with particular reference to qualia properties). The symmetric ring starting from the word "turn" in the sense "the leaves turn in Autumn" involves different properties: "turn, grow" is distinguished from "change" by specifying that the timescale is gradual, while "discolour" specifies which attribute is to change; "turn" in the above sense inherits both properties of gradual timescale and colour attribute.

In the remaining three cases of symmetric rings, the gloss for the initial synset contains the word "or", conveying an ambiguity. The two hypernyms in each case are in fact hypernyms or synonyms of the respective two meanings, and the grandparent is indeed a common ancestor. Splitting the ambiguous synsets to remove the ring would improve consistency.

We conclude that in 7 out of 8 instances, rings among verbs can be corrected and in the remaining case ("turn", above) the ring and the dual inheritance are justified. The question then arises as to why there should only be one case of a semantically justifiable ring. The answer to this question is sought through an investigation of the remaining 23 cases of dual inheritance among verbs, where there is no ring. 


\subsection{Dual inheritance}

Table 2 shows the results of a review of the semantic relations encoded as hypernymy in a dual inheritance context. 3 hypernyms have an ambiguous troponym whose gloss contains the word "or", and 2 hypernyms are themselves ambiguous with glosses containing "or".

\begin{tabular}{|l|r|}
\hline Correct: Telic quale & 5 \\
\hline Correct: Formal quale & 4 \\
\hline Otherwise correct hypernym & 10 \\
\hline $\begin{array}{l}\text { Hypernym of 1 sense of ambiguous } \\
\text { troponym }\end{array}$ & 3 \\
\hline No near relation & 4 \\
\hline Synonym of other hypernym & 2 \\
\hline Temporal inclusion & 4 \\
\hline Backward presupposition & 1 \\
\hline Ambiguous hypernym & 2 \\
\hline Reflexive cause of sister term & 1 \\
\hline Sister term & 2 \\
\hline $\begin{array}{l}\text { Reverse proper inclusion of 1 sense } \\
\text { of ambiguous troponym }\end{array}$ & 1 \\
\hline $\begin{array}{l}\text { Intransitive form of transitive tro- } \\
\text { ponym }\end{array}$ & 1 \\
\hline Synonym & 1 \\
\hline $\begin{array}{l}\text { Non-reflexive form of reflexive } \\
\text { troponym }\end{array}$ & $\mathbf{4 6}$ \\
\hline Transitive cause & 1 \\
\hline Troponym & 1 \\
\hline Literal sense of metaphor & \\
\hline TOTAL & \\
\hline$\%$ correct & \\
\hline
\end{tabular}

Table 2. Verb hypernyms involved in dual inheritance without rings

The cases of proper inclusion and backward presupposition should be encoded as entailments according to Fellbaum's (1998) specification and causes also should be encoded as such.

\begin{tabular}{|l|l|l|}
\hline Word forms & Formal quale & Telic quale \\
\hline $\begin{array}{l}\text { date, } \\
\text { date stamp }\end{array}$ & stamp & date \\
\hline $\begin{array}{l}\text { assemble, } \\
\text { piece }\end{array}$ & $\begin{array}{l}\text { join, bring } \\
\text { together }\end{array}$ & make, create \\
\hline $\begin{array}{l}\text { execute, put } \\
\text { to death }\end{array}$ & Kill & $\begin{array}{l}\text { punish, pe- } \\
\text { nalize }\end{array}$ \\
\hline Carve & Cut & shape, form \\
\hline Sing & Utter & $\begin{array}{l}\text { interpret, } \\
\text { render }\end{array}$ \\
\hline
\end{tabular}

Table 3. Dual inheritance justified by qualia
Table 3 shows details of those 4 cases where dual inheritance can be justified in terms of inheritance of two different qualia (Amaro et al., 2006). The fifth example, in italics, is not as encoded in WordNet: "sing" (intransitive) is given as a hypernym of "sing" (transitive), which is otherwise synonymous, consistent with the encoding of "eat" mentioned above. The other hypernym, "interpret, render" is necessarily transitive. The hypernym of "sing" (intransitive) is given as "talk, speak", which is really a sister term whose common hypernym would be "utter" (Miller \& Johnson-Laird, 1976), which represents the formal quale, while "interpret, render" represents the telic quale. So there is an underlying dual inheritance of different qualia properties.

The only cases of dual inheritance which can be considered legitimate are those where different but compatible properties are inherited. While this handful of examples has been identified, there are no doubt many more which could be discovered (Amaro et al., 2006). The encoding of these might well result in further justifiable rings.

\section{Anomalies relating to Categories}

\subsection{Isolators}

1593 examples were found of isolators among verbs and 2527 among nouns. These results approximate to those of Liu et al. (2004), who found 1551 verb isolators and 2654 noun isolators in WordNet 2.0. A review of the semantic relations was undertaken on a sample of 41 pairs of troponym and hypernym in different categories among verbs (table 4).

\begin{tabular}{|l|r|}
\hline Correct hypernymy & 26 \\
\hline $\begin{array}{l}\text { Troponym is true troponym of one } \\
\text { meaning of ambiguous hypernym }\end{array}$ & 1 \\
\hline Hypernym is cause of troponym & 2 \\
\hline $\begin{array}{l}\text { Troponym is true troponym of cause } \\
\text { of hypernym }\end{array}$ & 2 \\
\hline $\begin{array}{l}\text { Hypernym temporally includes tro- } \\
\text { ponym }\end{array}$ & 1 \\
\hline $\begin{array}{l}\text { Hypernym is backward presupposi- } \\
\text { tion of troponym }\end{array}$ & 1 \\
\hline Synonymous & 5 \\
\hline Metaphor & 1 \\
\hline No near relation & 2 \\
\hline TOTAL & $\mathbf{4 1}$ \\
\hline \% correct & $\mathbf{6 3 \%}$ \\
\hline
\end{tabular}

Table 4: Semantic relations among isolator samples 
Again we find cases of causation and entailment encoded as hypernymy. In most cases the hypernymy relation is correct, while the synsets belong to different categories. This arises inevitably because the WordNet verb categories are, for the most part, not mutually exclusive. In only one sample were the categories found to be mutually exclusive (change and stative). 14 out of the 82 synsets were judged not to belong to the category to which they are assigned, while 17 synsets do belong to the other's category.

The majority of WordNet's verbal semantic categories represent overlapping semantic fields. It is not therefore surprising that the isolator phenomenon occurs and this does not necessarily imply an error. The only categories which could be considered not to overlap are stative with change and creation, and weather with most of the other semantic fields.

A more detailed examination was made of a sample of 76 cases of isolator, where the hypernym and troponym categories could be considered mutually exclusive. The incorrect examples in table 5 are typical of the kinds of errors we have already seen in the encoding of WordNet relations.

\begin{tabular}{|l|l|}
\hline Valid relation & 55 \\
\hline No near relation & 5 \\
\hline $\begin{array}{l}\text { Hypernym is reflexive cause of } \\
\text { troponym }\end{array}$ & 1 \\
\hline $\begin{array}{l}\text { Indeterminate (ambiguous syn- } \\
\text { set) }\end{array}$ & 6 \\
\hline Syntactic alternation & 2 \\
\hline $\begin{array}{l}\text { Hypernym is backward presup- } \\
\text { position of troponym }\end{array}$ & 2 \\
\hline $\begin{array}{l}\text { Hypernymy only because of mis- } \\
\text { leading gloss }\end{array}$ & 1 \\
\hline Sister terms of tro- & 1 \\
\hline $\begin{array}{l}\text { Hypernym is reflexive } \\
\text { ponym }\end{array}$ & 1 \\
\hline Synonymous & 1 \\
\hline $\begin{array}{l}\text { Troponym is transitive cause of } \\
\text { true troponym }\end{array}$ & $\mathbf{7 6}$ \\
\hline TOTAL & $\mathbf{7 2 \%}$ \\
\hline \% correct
\end{tabular}

Table 5. Semantic relations among isolator samples involving mutually exclusive categories

Some 53 synsets $(34.9 \%)$ were considered to be in the correct category. Change has been used indiscriminately to include both causative and inchoative alternations, while both creation and stative have been used for the causative alternations and stative has also been used for some of the inchoative alternations. By transferring all the inchoative alternations to change, and all the causative alternations to creation, some of the category mismatches disappear.

Some of the verbs in this dataset have generic meanings like doing and happening, which do not fit into any of the WordNet categories. Creating categories for these verbs might eliminate more anomalies.

\subsection{Theoretical Basis of Categories}

In theory, WordNet nouns and verbs are arranged as a set of hierarchies (Fellbaum 1998), each with an unique beginner, but multiple inheritance is not ruled out, particularly where the second hypernym of a synset is in a different semantic category. The semantic categories in WordNet are based, according to Fellbaum (1998) on a standard work on psycholinguistics (Miller \& Johnson-Laird, 1976). The latter discusses in detail verbs of motion, possession, vision (WordNet perception) and communication, which are the basis for the corresponding WordNet categories. Other semantic fields mentioned are contact, bodily activity (WN body), thought (WN cognition) and affect (WN emotion). Miller \& Johnson-Laird, (1976) acknowledge that these categories overlap, but WordNet does not allow a verb to belong to more than one category. No theoretical basis has been found for the remaining categories. Competition is subsumed by social, and consumption is subsumed by body. Weather would seem selfcontained, but change, creation and stative are not semantic fields at all. Stative belongs to the Aktionsart categorisation of verbs distinguishing it from verbs of activity, achievement and accomplishment, which is orthogonal to the categorisation of verbs into semantic fields (Vendler, 1967, Moens \& Steedman 1988, Amaro, 2006). Moreover, a verb can belong to more than one Aktionsart category, as these apply to verbs in contexts.

\subsection{Suggested Revision of Categories}

Among verbs, the level of arbitrariness and incorrectness of the WordNet categories seems greater than that of the relations. Whereas the theoretical basis for WordNet relations is consistent and the errors are failures to conform to the specification, in the case of categories, the theoretical basis is inconsistent, being, a compromise between more than one system of categorisation. 
Many anomalies arise because WordNet only allows a verb to belong to one category. It is impossible to encode semantic categories correctly on the basis of one category per verb. At least two semantic fields need to be allowed, plus at least one orthogonal category. Miller \& JohnsonLaird (1976) themselves point out that semantic components like cause and intention cross the boundaries between semantic fields as do operators like happen, do, act, possible and permissible.

\section{Top Ontology}

\subsection{Candidate Unique Beginners}

WordNet 3.0 has 559 verbs with no hypernym, spread over all categories. Of these, 225 have no troponyms either. A further 96 have a single troponym of which 80 have no further troponyms. This leaves 254 verbs which have no hypernym and more than 1 direct or indirect troponym, in contrast with the theoretical position that each verb category has at most a handful of unique beginners (Fellbaum, 1998). These 254 verbs will therefore be considered as candidate unique beginners.

More than one candidate was found in every category, the minimum being 5 for category 34 consumption. According to Fellbaum (1998), category 38 motion should have two unique beginners "expressing translational movement" and "movement without displacement" respectively. There are 17 other candidates in this category. Similarly category 40 , possession should have 3 unique beginners, representing the basic concepts "give", "take" and "have", whereas there are 12 other candidates.

Again, according to Fellbaum (1998) "Communication verbs are headed by the verb communicate but immediately divide into two independent trees expressing verbal and nonverbal (gestural) communication". There are 7 senses of "communicate" in WordNet 3.0 all of which have hypernyms. Fellbaum (1998) identifies a further subdivision between spoken and written language, but the only reference to "write" among these 254 synsets occurs in category 36 : creation. In fact category 32 communication has 18 candidates. There appears to be no connection between the theory and the practice here.

It is always possible to define a verb in terms of another verb with one or more arguments. This is a method of identifying hypernyms, which appears to have been used extensively, though inconsistently, in the construction of WordNet, using the glosses for semi-automatic hypernym generation. Full automation of such a technique would lead inevitably to the cycle scenario defined above. There have to be unique beginners in order to avoid this.

\subsection{Deriving a New Top Ontology}

On a dataset of this size, it is feasible to manually identify hypernyms for most of the synsets, though there is more than one possible solution in many cases. In some cases it is sufficient to provide a more generic verb or verbal phrase as hypernym, which already belongs to a hierarchy. In other cases a combination of a verb and one or more arguments (mostly involving an additional verb) is required in order to define the verb, in which case the syntactic main verb in the definition can be considered as the hypernym. Auxiliary verbs required by the definitions include one modal verb ("can") not included in WordNet.

The 254 synsets were manually annotated either with proposed hypernyms or with definitions in terms of verbs and arguments, without reference to semantic categories. In some cases auxiliaries act in conjunction with each other to form more complex definitions. The definitions are tentative, the objective being to demonstrate the possibility of generating a compact and consistent top ontology. However, there is scope for introducing more rigour by formalising the definitions along the lines of Jackendoff's $(1983,1990)$ lexical conceptual structures.

Table 6 shows the auxiliaries used in defining the candidate unique beginners. The first row represents verbs which can be defined as the passive of another verb, namely "hang" (be supported from above), "depend (on)" (be caused by) and "belong (to)" (be had by).

The next row but one represents transitive causation verbs e.g. "kill" (cause to die), "sensitize" (cause to sense), show (cause to see) etc. There is also negative causation, of which the most generic case is the verb "prevent". There are fewer, but a significant number of examples of reflexive causation, e.g. "look" (cause self to see) and more complex cases concerning possession: "give" (cause to have + cause self to not have), "take" (cause to not have + cause self to have).

There are a number of unique beginners which express starting or stopping e.g. "learn" (start to know), "get" (start to have), "become" (start to be) and "lose" (stop having).

Finally there are verbs which can be defined as the negation of other verbs: "displease" (not please), "reject" (not accept), "fail" (not suc- 
ceed), "lack" (not have), "refrain" (not do). Although "NOT" is not a verb, "doesn't/don't" is a verb which is generally substituted for "NOT", except in the infinitive. On these grounds "NOT" is not out of place in this list.

\begin{tabular}{|l|l|l|l|l|}
\hline Aux. & Syntax & n & Hyp. & Args. \\
\hline Be & done & 3 & happen & \\
\hline Can & do & 1 & & \\
\hline Cause & to do & 43 & make & \\
\hline Cause & self to do & 6 & make & self \\
\hline Feel & like doing & 2 & & \\
\hline $\begin{array}{l}\text { Keep } \\
\text { (repeat) }\end{array}$ & doing & 6 & do & again \\
\hline Start & doing & 11 & & \\
\hline Stop & doing & 8 & & \\
\hline $\begin{array}{l}\text { Tell (1) } \\
\text { (someone) } \\
\text { to do }\end{array}$ & 1 & tell (2) & $\begin{array}{l}\text { that you } \\
\text { must }\end{array}$ \\
\hline $\begin{array}{l}\text { Try } \\
\text { to do }\end{array}$ & 1 & will & $\begin{array}{l}\text { although } \\
\text { NOT } \\
\text { know } \\
\text { whether } \\
\text { can }\end{array}$ \\
\hline $\begin{array}{l}|l| \\
\text { NoT }\end{array}$ & $\begin{array}{l}\text { do } \\
\text { ries }\end{array}$ & 22 & & \\
\hline $\begin{array}{l}\text { Tell (2) } \\
\text { (someone) } \\
\text { that }\end{array}$ & 3 & cause & know \\
\hline Must & do & 1 & & \\
\hline Will & do & & \\
\hline
\end{tabular}

Table 6. Auxiliaries needed to define candidate unique beginners $($ Aux. $=$ auxiliary; $\mathrm{n}=$ number of candidates defined; Hyp. = proposed hypernym; Args. $=$ arguments)

In order to identify hypernyms from these definitions consistently, we take the auxiliaries, including "NOT", as the immediate hypernyms.

A second meaning of "tell" has been added to table 6 as this has not been subsumed. The hypernyms and arguments themselves introduce two more modal auxiliaries, "must" and "will" (in the Old English sense of volition).

The implementation of this approach to identifying hypernyms for candidate unique beginners, left 14 which could not be subsumed, (table 7). The two synonymous synsets in category 41 can be merged. Weather verbs of zero valency are subsumed by "happen".

Those auxiliaries used in the definitions which cannot be subsumed by a hypernym must be represented in the list of outstanding unique beginners. Of these, "feel", "start" and "stop" are already listed. The modals ("can", "must" and "will") and "NOT" need to be added to table 7 to complete a proposed new compact and consistent top ontology for verbs comprising 18 unique beginners.

There are relations between these concepts and the list could probably be further reduced. The modal verbs, together with "know" form a natural set of entailments for "do". The concept "do" compulsorily backwardly presupposes the combination of "can" and "will" ("able and willing"). "Will" may be underlain or superseded by "must". "Can" can mean either "is physically equipped to" or "knows how to" or both and corresponds to the operator possible in the schema of Miller \& Johnson-Laird (1976) referred to above, while "must" means NOT(permissible(NOT to)) using the operator permissible from the same schema.

\begin{tabular}{|l|l|l|}
\hline Synset ID & Word Forms & Cat. \\
\hline 200109660 & change & 30 \\
\hline 200339934 & happen, hap, go on & 30 \\
\hline 200594621 & know, cognize, cognise & 31 \\
\hline 200628491 & think, cogitate, cerebrate & 31 \\
\hline 201617192 & make, create & 36 \\
\hline 201712704 & perform, execute, do & 36 \\
\hline 201771535 & feel, experience & 37 \\
\hline 201831531 & move (non-translational) & 38 \\
\hline 201835496 & $\begin{array}{l}\text { travel, go, move (transla- } \\
\text { tional) }\end{array}$ & 38 \\
\hline 202106506 & perceive, comprehend & 39 \\
\hline 202367363, & act, move & 41 \\
\hline 202419073 & & 42 \\
\hline 202603699 & exist, be & 42 \\
\hline 202608347 & begin, start & 42 \\
\hline 202609764 & end, stop, finish, terminate \\
\hline Addendum & can & $\boldsymbol{n} / \boldsymbol{a}$ \\
\hline Addendum & must & $\boldsymbol{n} / \boldsymbol{a}$ \\
\hline Addendum & will & $\boldsymbol{n} / \boldsymbol{a}$ \\
\hline Addendum & NOT & \\
\hline
\end{tabular}

Table 7. New top ontology comprising outstanding unique beginners and modals

\section{Conclusion}

In the course of this study, one cycle has been found in WordNet and a number of "multi-parent relations" have been found to be unjustified. Over 500 instances have been found of "top-most synset not from the defined set" or "dangling uplinks" (Smrž, 2003). Many shortcomings have been found in the encoding of hypernymy between verbs, where the implementation fre- 
quently does not conform to the theory. In their present state, the encoded relations can only reliably serve to indicate that a relation exists between two verbs and not to elucidate what that relation is. One is left with the impression that many hypernyms have been encoded arbitrarily, whether manually or automatically, with little respect for distinctions between kinds of relation. The hierarchy could be improved by merging synonymous synsets and removing redundant relations and by adopting a consistent approach to causative/inchoative alternations. The only valid cases of dual inheritance are where different but compatible properties are inherited. Many more such relations could be encoded. The semantic categories are, for the most part, not mutually exclusive and lack a consistent theoretical basis. If semantic categories are required, then a verb needs to be allowed to belong to more than one. A new top ontology has been proposed which reduces the number of unique beginners from 254 to 18 with the aid of auxiliaries including modal verbs.

The issues identified can be addressed by building a new lexical database from the same sources, but there would be a very substantial overhead of applying manual corrections to the entire verb hierarchy or constructing an entirely new set of relations with a clear theoretical basis in a non-arbitrary manner, along the lines suggested by Guarino (1998). One possible alternative is to borrow the relational structure from another lexical database.

\section{Acknowledgement}

Thanks are due to Dr. S. H. S. Wong and Dr. C. Buckingham of Aston University for suggestions regarding the presentation of these findings.

\section{References}

Amaro, R. 2006. WordNet as a base lexicon model for computation of verbal predicates. Proceedings of the Third Global WordNet Conference, 22-26 January 2006, Jeju Island, Korea.

Amaro, R., Chaves, R. P., Marrafa, P. \& Mendes S. 2006. Enriching Wordnets with new Relations and with Event and Argument Structures. Seventh International Conference on Intelligent Text Processing and Computational Linguistics, Mexico City, 2006, $28-40$.

Banerjee, S. \& Pedersen, P. 2003. Extended Gloss Overlaps as a Measure of Semantic Relatedness. Proceedings of the Eighteenth International Joint
Conference on Artificial Intelligence, Acapulco, Mexico, August, 2003.

Fellbaum, C. 1998. A Semantic Network of English Verbs, Fellbaum, C. (ed.), WordNet: An Electronic Lexical Database, Cambridge, Massachusetts, MIT Press, 69-104.

Guarino, N., 1998. Some ontological principles for designing upper level lexical resources. In: Proceedings of the First International Conference on Language Resources and Evaluation, LREC 98.

Jackendoff, R., 1983, Semantics \& Cognition, Cambridge, Massachusetts, MIT Press.

Jackendoff, R., 1990, Semantic Structures, Current Studies in Linguistics Series, Cambridge, Massachusetts, MIT Press.

Lesk, M., 1986. Automatic sense disambiguation using machine readable dictionaries: How to tell a pine cone from a ice cream cone. In Proceedings of SIGDOC'86,

Liu, Y., Jiangsheng Y., Zhengshan W. \& Shiwen Y. 2004. Two Kinds of Hypernymy Faults in WordNet: the Cases of Ring and Isolator. Proceedings of the Second Global WordNet Conference. Brno, Czech Republic, 20-23 January 2004. 347-351.

Miller \& Johnson-Laird. 1976. Language \& Perception, Cambridge University Press, Chapter 7, 524689.

Moens, M. \& Steedman, M. 1998. Temporal Ontology and Temporal Reference, Computational Linguistics, 14 (2), June 1988, 15-28.

Pustejovsky, J., 1991. The Generative Lexicon, Computational Linguistics, 17, (4), 409-441

Smrž, P. 2004. Quality Control for Wordnet Development. Proceedings of the Second Global WordNet Conference. Brno, Czech Republic, 20-23 January 2004. 206-212.

Various, 2004. Design and Development of a Multilingual Balkan Wordnet Balkanet, IST-200029388, WP8: Restructuring Improvement WordNets, Deliverable D8.1: Restructuring WordNets for the Balkan languages, Project Report, European Commission

Vendler, Z., 1967, Verbs \& Times, Vendler, Z. (ed.), Linguistics in Philosophy, Cornell University Press, Ithaca \& London, 97-121,

Vossen, P. 2002. EuroWordNet General Document, Project Report. 